Relations industrielles

Industrial Relations

\title{
La participation de la collectivité à une planification économique, Cahier de l'I.C.E.A., Montréal, no 3, février 1967, 144 pages.
}

\section{Pierre Dionne}

Volume 22, numéro 2, 1967

URI : https://id.erudit.org/iderudit/027791ar

DOI : https://doi.org/10.7202/027791ar

Aller au sommaire du numéro

Éditeur(s)

Département des relations industrielles de l'Université Laval

ISSN

0034-379X (imprimé)

1703-8138 (numérique)

Découvrir la revue

Citer ce compte rendu

Dionne, P. (1967). Compte rendu de [La participation de la collectivité à une planification économique, Cahier de l'I.C.E.A., Montréal, no 3, février 1967, 144 pages.] Relations industrielles / Industrial Relations, 22(2), 301-301.

https://doi.org/10.7202/027791ar

Tous droits réservés @ Département des relations industrielles de l'Université Laval, 1967
Ce document est protégé par la loi sur le droit d'auteur. L’utilisation des services d'Érudit (y compris la reproduction) est assujettie à sa politique d'utilisation que vous pouvez consulter en ligne.

https://apropos.erudit.org/fr/usagers/politique-dutilisation/ 
beaucoup d'attention. Néanmoins, tous ceux qui s'intéressent aux problèmes de l'automation y puiseront des données fondamentoles sur cette question en U.R.S.S.

\section{Pierre DIONNE}

Travail et automation - Etudes de cas sur l'évolution technique: tableaux analytiques, Bureau international du travail, cahier no 2 , Genève 1965, 89 pages.

L'une des première méthodes d'investigation utilisées aux fins de connaître les répercussions de l'automation, est l'étude de cas.

- Limitée ò une entreprise, ou encore à un groupe d'entreprises d'une même branche d'activité ou d'un même secteur, l'étude de cas a pour objet d'examiner en détail les conséquences de telle ou telle innovation technique dans l'entreprise, la branche d'activité ou le secteur considéré.

Le présent cahier présente sous forme de tableaux l'analyse de cent soixante études de cas, menée dons quatorze pays auprès d'entreprises de vingt-neuf branches d'activité

Du fait même de sa nature, l'analyse ne s'étend pas aux résultats auxquels ont pu conduire les études de cas considérées, pas plus qu'elle ne permet de tirer des conclusions. On peut penser toutefois que ce cahier, tout en fournissant aux chercheurs un instrument de référence, sera de quelque utilité pour l'établissement des programmes d'investigations futures.

\section{Pierre DIONNE}

\section{La participation de la collectivité à une} planification économique, Cahier de II.C.E.A., Montréal, no 3, février 1967, 144 pages.

Les pages de ce cahier ne contiennent pas des études sur la planification en tant que telle, mais bien un ensemble de réflexions sur la participation des collectivités à une pianification démocratique et efficace.

C'est à un effort de démystification que les outeurs tentent $d$ 'arriver par une approche concrète et pragmatique des problèmes. Le présent cahier ne s'adresse donc pas à des spécialistes de la planification, mais « au citoyen désireux d'être mieux informé et documenté sur la question, afin d'apporter ou meilleur de sa connaissance sa quote part de participation $\gg$.

$\mathrm{Ce}$ cahier se divise en deux parties. La première tente d'éclairer la notion même de planification démocratique, les modalités de consultation et la nature des orgonismes dont nous disposons au Canada et au Québec. La seconde partie décrit à partir des expériences du B.A.E.Q. et du Lac-St-Jean, le processus ou les méthodes utilisées, compte tenu de certains conditionnements et facteurs d'influence.

Bref, les textes que contient ce cahier, tendent à démontrer qu'aucun gouvernement ne peut se passer des corps intermédiaires dans l'édification de plans socio-économiques.

\section{Pierre DIONNE}

La Ville: phénomène économique, par Jean Rémy, Editions Vie Ouvrière, Bruxelles, 1966, 297 pages.

Cet ouvroge tente d'examiner la signification économique de la ville, surtout de la ville de grande dimension et d'en étudier le rôle accru tant pour le bien-être des populations que pour l'organisation efficace de lc production des divers biens.

Pour l'auteur le développement urbain, s'il s'opère adéquatement, doit valoriser l'urbanisation. La théorie de la croissance économique doit reconnaître le rôle des facteurs urbains et, la politique économique doit organiser la croissance urbaine de manière à réduire ou minimum les coûts qui en résultent.

Dans un premier temps, Jean Rémy analyse la signification de la juxtaposition spatiole - méthodes, étapes, causes - et le contenu des économies d'agglomération du point de vue de l'entrepreneur. Ensuite, il reprend les économies d'agglomération du point de vue du consommateur final.

L'auteur continue son étude en donnant le point de vue du sociologue sur la ville dans l'organisation et la croissance d'un ensemble social.

Dans un deuxième temps, l'analyse porte sur les diséconomies engendrées par l'agglomération et l'organisation de l'espace comme moyen d'y remédier.

Enfin, l'étude se termine en envisageant le développement urbain comme objectif d'une politique économique. 